\title{
Influence of errors in prescriptions on the security of medicine
}

\author{
K. Puke ${ }^{1}$, K. Spalva ${ }^{1}$, and I. Urtane ${ }^{2}$ \\ ${ }^{1}$ University of Latvia, Riga, Latvia \\ ${ }^{2}$ Rīga Stradiņš University, Riga, Latvia
}

\begin{abstract}
All types of medication errors including missed doses, incorrect dosage forms, time intervals, and routes are essential encumbrances for qualitative pharmaceutical care and security of medicine [1]. Problems related to prescription errors are common in the healthcare profession, and are responsible for significant increase in costs, cases of morbidity and mortality [2]. The aim of the study was to analyze the common errors in prescriptions which were received in pharmacies and their effect on the security of medicine. Retrospective study was conducted between December 2013 and January 2014 in the pharmacy of Riga, Latvia. Prescriptions were analyzed to identify errors in Inscriptio, Praescriptio and the Signatura part. Of 200 prescriptions, only 14 (7\%) were filled correctly according to the legislative requirements in Latvia. The most common drug therapeutic class in the prescriptions was non-steroidal anti-inflammatory drugs (NSAID) and other analgesics $(21.1 \%)$. Unclear handwriting was observed in more than one third of all studied prescriptions $(n=72 ; 36.0 \%)$. Mean age values of physicians were higher, but not significantly different, in the unclear compared to clear prescriptions, $59.5 \pm 8.5$ vs. $57.8 \pm 10.6$, respectively $(\mathrm{p}=0.253)$. Omission of the quantity of drug in the prescription part was the most frequent type of the error $(n=112,56.0 \%)$. High level of incorrect prescriptions was found during the period of study in the pharmacy. Overall, approximately $27 \%$ of prescriptions had significant failures, which could negatively affect therapeutic effect and safety of drug use.
\end{abstract}

\section{Introduction}

Prescriptions are the primary means of communication between prescribers and pharmacists concerning instructions on medication. Unfortunately, a large percentage of outpatient paper prescriptions contain one or more errors, resulting in millions of prescriptions with the potential to harm patients [3,4]. An estimated 1 of every 131 outpatient deaths is attributable to medication errors [5]. Prescribing errors are a common cause for preventable medication errors and adverse drug events in primary care [6]. Most commonly observed prescription errors include incorrect selection of medication for the patient including the dose, quantity, indication, or prescribing a contraindicated medication [7]. Community pharmacists have an important role in detecting, preventing, and solving prescription problems, which, if left unresolved, may pose a risk of harming the patient [8]. Pharmacists can intervene to eliminate or minimize these risks, often by contacting the prescriber with suggestions to change the medication [9].

The aim of the study was to analyze the common prescription errors noticed in prescriptions received by the pharmacy and their effect on the security of medicine. 


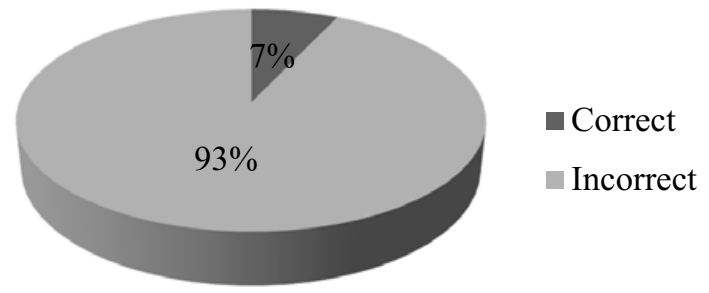

Figure 1. Characteristics of Prescriptions.

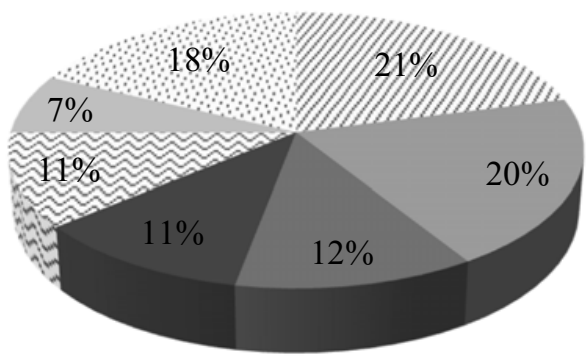

\% NSAID

Antibiotics

GI

- Cardiovascular

¿Vasodilators

Diuretics

$\therefore$ Others

Figure 2. Therapeutic Classes of Drugs.

\section{Materials and methods}

Retrospective study was conducted between December 2013 and January 2014 in the pharmacy of Riga, Latvia. Prescriptions were analyzed to identify errors in Inscriptio, Praescriptio and the Signatura part. Data analysis was developed using SPSS ver.16 and Microsoft Excel.

\section{Results}

Of 200 prescriptions only $14(7 \%)$ were filled correctly according to the legislative requirements in Latvia (Fig. 1).

Frequency of physicians who had signed selected prescriptions was the following: family doctors $(n=129,64.5 \%)$, internists $(n=18,9.0 \%)$, surgeons $(n=12,6.0 \%)$. The most common drug therapeutic classes in the prescriptions were non-steroidal anti-inflammatory drugs (NSAID) and other analgesics (21.0\%), antibiotics (20.0\%), GI tract drugs (12.0\%) (Fig. 2).

Unclear handwriting was observed in $72(36.0 \%)$ prescriptions. Mean age values $( \pm$ SD) of physicians were higher but not significantly different in the unclear, compared to clear prescriptions: $59.5 \pm 8.5$ vs. $57.8 \pm 10.6$ ( $\mathrm{p}=0.253$ ). In the inscription part, errors were found in 86 prescriptions, most commonly due to lack of the name of the patient $(n=39,19.5 \%)$, address $(n=31,15.5 \%)$, rarely lack of the surname and personal identification code of the patient $(n=4,2.0 \%)$. Omission of the quantity of drug in the prescription part was the most frequent type of the error $(n=112,56.0 \%)$, with dose errors being the second most frequent $(\mathrm{n}=71,35.5 \%)$ (Table 1$)$.

Errors due to the lack of quantity and dose of the drug simultaneously were more frequent compared to prescriptions with lack of quantity but with correct dose of the drug $(n=68$, $60.7 \%$ vs. $\mathrm{n}=36,32.1 \% ; \mathrm{p}<0.01$ ) (Fig. 3). 
Table 1. Number of Errors (N) in Praescriptio.

\begin{tabular}{|l|c|c|c|}
\hline Characteristic of drug forms & Correct & Not exact & Incorrect \\
\hline Form & 72 & 41 & 87 \\
\hline Quantity & 62 & 26 & 112 \\
\hline Dose & 117 & 12 & 71 \\
\hline
\end{tabular}

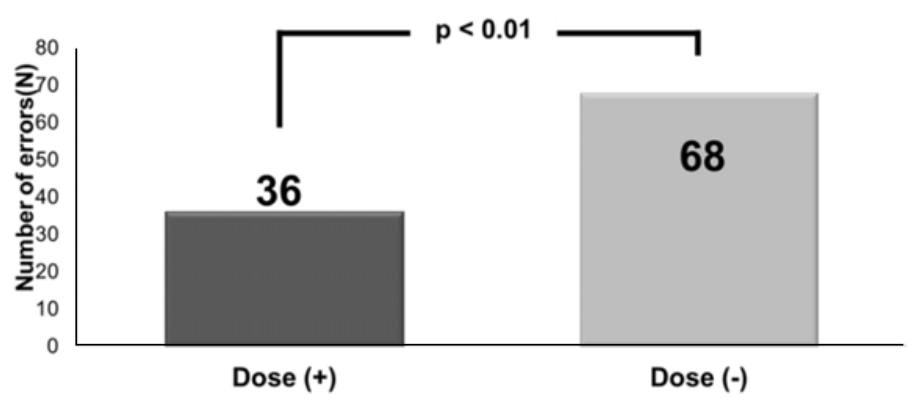

Figure 3. Errors of Prescribed Drug Quantity and Dose Simultaneously.

In prescriptions, errors in both dose and use of medication were found statistically significantly less frequently $(8.0 \%)$ compared with prescriptions, where the dose was not pointed, but the use of the medication was indicated $(24.7 \%)(\mathrm{p}<0.01)$.

The lack of dose was found for the following pharmacological groups: antibiotics (19.1\%), NSAIDs (16.9\%), vasodilators $(14.6 \%)$, cardiovascular and GI tract drugs $(12.4 \%)$ and antihistamines (11.2\%).

In 33 prescriptions $(n=16.5 \%)$ we observed failures in Signatura part due to the lack of regime of drug use.

\section{Discussion}

Pharmacists have received a large proportion of prescriptions which have not been properly written. This fact indicates that attention to the correct information about patients and prescribed medications should be enhanced.

Approximately $1 / 3$ of the prescriptions, analyzed during the study, were written by family doctors. A similar proportion of family doctors was also found elsewhere in the studies on the prevalence of prescription errors [10].

Dividing the prescribing doctors according to the age of two selected groups $(<50$ and $>$ 50 years), it was concluded that the age did not directly affect unclear handwriting, respectively, increasing physician's age was not related to increase in the number of cases of unclear handwriting.

More frequently unclear handwriting was observed among such specialists as ENT doctors, surgeons and gynaecologists. This observation could be explained by the fact that in family doctor's practices nurses often write prescriptions according to doctor's instructions.

From our study the most commonly prescribed pharmacological groups of prescribed medications were NSAIDs (21.1\%) and antibiotics (20.0\%), which was similar to the Nadiya et.al study in Oman, where NSAIDs were prescribed in $35.11 \%$ and antibiotics in $18.22 \%$ of prescriptions [11]. According to the literature review the dose corresponded to type A errors. Type A errors in our study were $48.0 \%$. These findings were similar in Bahrain (31.0\%) [11] and Oman $(22.11 \%)$ studies [12]. In other study, that analyzed mistakes in prescriptions in 
Estonia, Norway and Sweden, the dosage form and dosage were defined as errors with clinical significance [13].

According to results of our study, the dose in prescriptions more often was not indicated for antibiotics (19.1\%), NSAIDs (16.9\%) and vasodilators (14.6\%). Researchers in the study from Saudi Arabia had not found similar results in prescriptions of antibiotics where lack of dose was observed in $18 \%$ cases [14]. Inaccuracies in dosing regime in Bahrain study [11] were the same in percentage value as in our study $-14 \%$, while in Oman study they were more frequent $-18.4 \%$ [12].

In our study administrative errors were in $40.8 \%$ of prescriptions, similarly with Norway $38.9 \%$ and Estonia 49.5\% [13].

In Latvia, we have a pilot study with e - prescription system. It can lead to major changes in the pharmacist's and the doctor's daily work, also by affecting filling requirements in prescriptions.

\section{Conclusions}

1. Overall, approximately $27 \%$ of prescriptions had significant failures, which can negatively affect therapeutic effect and safety of drug use.

2. High level of incorrect prescriptions was found during the period of study in the pharmacy.

3. The most common errors in the Praescriptio part were those with negative influence on the pharmaceutical care and the security of medicine.

\section{References}

[1] Guchelaar, Henk-Jan.Hadewig B. et.al. Medication Errors. September 2005, 65, Issue 13, pp 1735-1746a.

[2] Calligaris, L. Panzera, A. et.al. Errors and omissions in hospital prescriptions: a survey of prescription writing in a hospital. BMC Clinical Pharmacology. May 2009, 9: 9.

[3] Hawksworth GM, Corlett AJ, Wright DJ et al. Clinical pharmacy interventions by community pharmacists during the dispensing process. Br J Clin Pharmacol.1999; 47: $695-700$.

[4] Devine EB, Wilson-Norton JL, Lawless NM et al. Characterization of prescribing errors in an internal medicine clinic. Am J Health-Syst Pharm. 2007; 64: 1062-70.

[5] Kohn LT, Corrigan JM, Donaldson MS. To err is human: building a safer health system. Washington, DC: National Academy Press; 1999.

[6] Dean B, Barber N, Schachter M. What is a prescribing error? Qual Health Care. 2000; 9(4): 232-237.

[7] Kuo GM, Phillips RL, Graham D, Hickner JM. Medication errors reported by US family physicians and their office staff. Qual Saf Health Care. 2008; 17(4): 286-290.

[8] Council of Europe. Creation of a better medication safety culture in Europe: Building up safe medication practices. Expert Group on Safe Medication Practices (P-SP-PHSAFE) (2006). Available from http://www. coe.int/t/e/social_cohesion/socsp/Medication\%20safety\%20culture\%20report\%20E.pdf (Accessed April 14, 2011).

[9] Baciu A, Stratton K, Burke SP, eds. The Future of Drug Safety. Promoting and Protecting the Health of the Public. Washington: The National Academies Press; 2007.

[10] Kennedy, A.K., Littenberg, B., Callas, P.W., et.al. Evaluation of a modified prescription form to address prescribing errors. Am J Health-Syst Pharm, 2011, Jan 15, 68. p.151. 
[11] Khaja, K.A., Ansari, T.M., Sequeira, R.P. An evaluation of prescribing errors in primary care in Bahrain. Int J Clin Pharmacol Ther. 2005 Jun; 43(6): 294-301.

[12] Shahaibi, N.M, Said, L.S., Kini, T.G., et.al. Identifying errors in handwritten outpatient prescriptions in Oman. J Young Pharmacists, 2012; 4: 267-72.

[13] Volmer, D., Haavik, S., Ekedahl, A. Use of a generic protocol in documentation of prescription errors in Estonia, Norway and Sweden. Pharmacy Practice (Internet), 2012 Apr-Jun; 10(2): 72-77.

[14] Jeraisy, M.I., Alanazi, M.Q., Abolfotouh, M.A. Medication prescribing errors in a pediatric inpatient tertiary care setting in Saudi Arabia. BMC Research Notes. 2011, 4: 294. 